OUVERTURE 


\title{
ÉTHOLOGIE ET CRIMINOLOGIE CLINIQUE : DEBUYST AVEC DEMARET POUR UNE ÉTHIQUE DE L'ADAPTATION
}

Christophe ADAM*

Jérôme ENGLEBERT**

\author{
"Quel est ton but en philosophie? \\ Montrer à la mouche l'issue par où s'échapper \\ de la bouteille à mouches ». \\ Ludwig Wittgenstein, \\ Recherches philosophiques, 1953, p. 309.
}

RÉSUMÉ Cet article noue un dialogue entre deux figures qui ont chacune un lien décisif avec l'éthologie (animale et humaine). Albert Demaret et Christian Debuyst, qui ne se connaissaient pas personnellement, proposent une réflexion d'une grande finesse, clinique et épistémologique, nous semblant ouvrir la voie à des perspectives de compréhension inédite pour la criminologie clinique. Dans un premier temps, nous traitons de la façon dont Debuyst mobilise l'éthologie dans une visée épistémologique, alors qu'un deuxième mouvement est consacré aux rapports que Demaret propose et observe entre

* Université libre de Bruxelles

** Université de Liège 
psychiatrie et éthologie animale. Dans une troisième partie, nous concrétisons ce dialogue entre ces deux perspectives pionnières, de manière à expliciter le gain d'intelligibilité que révèle la mise en contraste de leur pensée. En toile de fond, ce sont les processus d'adaptation mobilisés dans le champ de la criminologie clinique que nous cherchons à rendre explicites et à reconsidérer.

MoTS-CLÉS ethologie, criminologie, psychiatrie, adaptation, éthique.

ABSTRACT This article establish a dialogue between two figures who each have a crucial link with ethology (animal and human). Albert Demaret and Debuyst Christian, who does not know personally, offers a precious clinical and espitemological reflection, we pretend to open the way for new perspectives for understanding of clinical criminology. First, we discuss how Debuyst mobilizes ethology an epistemological referred while a second movement is dedicated to reports Demaret and offers observed between psychiatry and animal ethology. In the third part, we are delivering the dialogue between these two pioneering perspectives to explain the intelligibility gain that reveals their contrasted thinking. Finally, we wanted to highlight the process of adaptation mobilized in clinical criminology field.

KEYWORDS ethology, criminology, psychiatry, adaptation, ethics.

\section{Introduction}

Le projet de cet article est né d'une rencontre entre ses auteurs, dont chacun peut se réclamer d'un héritage intellectuel fécond. En effet, sans le savoir d'emblée, nous partageons une référence à l'éthologie, respectivement par les apports de deux grandes figures, Albert Demaret et Christian Debuyst, qui ne se connaissaient pas personnellement. En 1986, Debuyst affirme que son intention n'est pas de faire usage d'études éthologiques en vue d'une meilleure connaissance des comportements comme cela a été proposé 
en 1979 par Demaret, pour le domaine des maladies mentales (Debuyst, 1896, p. 311). Partant, nous avons voulu construire un dialogue entre eux - prolongeant celui qui, en amont et en aval, nous relie tous les deux -, en vue de montrer la puissance heuristique et la complémentarité de leurs références à l'éthologie, afin de penser cliniquement tantôt la psychopathologie, tantôt la criminologie ainsi que les liens intrinsèques qui unissent ces deux disciplines.

Dans un premier temps, nous traiterons de la façon dont Debuyst mobilise l'éthologie dans une visée épistémologique. Un deuxième mouvement sera consacré aux rapports que Demaret propose et observe entre psychiatrie et éthologie. Dans une troisième partie, nous nouerons un dialogue entre ces deux perspectives pionnières, de manière à expliciter le gain d'intelligibilité que révèle la mise en contraste de leur pensée.

\section{1. Éthologie et épistémologie de la criminologie : l'apport de Christian Debuyst}

Contrairement à ce que l'on pourrait penser à première vue, l'ouvrage de Christian Debuyst ${ }^{1}$, Modèle éthologique et criminologie (1985), ne fait pas strictement référence à l'éthologie, définie comme l'étude scientifique du comportement animal en milieu naturel. Pour comprendre le sens profond du projet de l'auteur, il conviendrait de le resituer dans l'architecture argumentative d'un texte qui trouve ses matériaux théoriques de construction dans quatre univers distincts, lesquels sont autant d'étapes révélant un emboitement successif : l'épistémologie des sciences, l'éthologie qui nous occupe ici, la reprise des travaux de son maître, le psychiatre et criminologue Étienne De Greeff - plus particulièrement sa théorie des instincts (De Greeff, 1947) - et, enfin, la psychologie sociale. Dans le cadre de cet article, nous nous limiterons au développement de quatre points - qui ne se recoupent qu'en partie avec les quatre jalons que nous venons d'annoncer. Après une formulation de la thèse épistémologique de Debuyst (1.1.), nous nous attacherons à préciser le gain issu de la référence à l'éthologie (1.2.). Ensuite, nous évoquerons le prolongement récent de ces travaux par la référence
1 Christian Debuyst est professeur émérite de l'UCL depuis 1990. Juriste, criminologue, psychologue, il est l'un des piliers de l'Ecole de Louvain en criminologie clinique et une figure importante dans l'évolution des savoirs en criminologie. Après avoir réalisé sa thèse de doctorat à partir de sa pratique de psychologue à la prison centrale de Louvain, il s'est fait connaître pour ses perspectives critiques face aux savoirs positivistes et aux tenants de la théorie de la personnalité criminelle. Aujourd'hui, il consacre ses travaux à l'art et à l'épistémologie clinique. 
à Wittgenstein (1.3.) qui nous paraît représenter une reprise sous un autre jour de la thèse de 1985. Enfin, nous esquisserons les tout nouveaux éléments de réflexion inaugurant le domaine de l'éthique (1.4.) que nous avons également pu saisir dans les intentions de l'auteur et qui nous serviront à élaborer notre proposition suggérant, sur fond d'adaptation clinique, un passage d'une «éthologie animale » à une «éthique de l'humain».

\subsection{La thèse épistémologique}

De manière générale, Debuyst nous met en garde concernant la portée et les valeurs de nos démarches de connaissance ainsi que les modèles théoriques sur lesquels elles se fondent. Il formule le problème en évoquant les grilles de lecture l'éthogramme cher à une certaine tradition de l'éthologie - à travers lesquelles nous percevons le réel. Il examine ainsi la manière dont nous prenons en compte les réalités et, particulièrement, les réalités délinquantes. Selon lui, l'objet délinquance, de par son inévitable impact affectif et émotionnel, est susceptible de mener à de puissantes déformations dans les processus de connaissance. Il faut préciser qu'aucune forme de connaissance n'y échappe : qu'elle soit celle de l'homme de la rue, de l'homme scientifique ou de l'homme délinquant lui-même. Dans une perspective universaliste, tous sont animés des mêmes mécanismes. Le fait d'avoir commis un acte posant problème au groupe social place le sujet dans un état d'alerte ou, s'il se sent agressé, peut le conduire à une réaction de défense. Ainsi, la grille de lecture du comportement problématique se voit orientée parce que le groupe se sent agressé, ce qui pourra légitimer une réaction de contre-agression, au sens d'une défense justifiant des attitudes de rejet de l'acte et de son auteur, considérés comme intolérables et inacceptables. D'un autre côté et pour des raisons identiques, la fascination pour la délinquance ou la « sympathie » pour les délinquants - notamment à travers des figures héroïques - peut témoigner d'une forme de connaissance tout aussi tendancieuse.

Sans rendre compte de la complexité d'ensemble développée dans Modèle éthologique et criminologique, ouvrage important pour l'épistémologie de la criminologie, nous allons 
détailler la référence à l'éthologie en ce qu'elle nous permettra de nourrir le dialogue au cœur du projet du présent article.

\subsection{La référence éthologique : une lecture utilitaire du réel à partir de la tique}

Lorsque Debuyst se tourne vers l'éthologie, c'est d'abord pour montrer que la connaissance participe d'une lecture utilitaire du réel permettant d'avoir prise sur celui-ci en vue d'une certaine efficacité. Cette dernière est liée à la sélectivité dans la connaissance, au sens où nous ne retenons que ce qui assure nos propres intérêts et la réalisation de nos objectifs. La référence à l'éthologie prend place dans cet argument du «tri sélectif» constitutif de la lecture utilitaire du réel. Ce matériau théorique permet à Debuyst de démontrer ce que la connaissance de la réalité doit à une sensibilité élective et préétablie à certains stimuli servant de signaux et d'indications pour mieux agir dans le réel. C'est donc à travers cette grille préalable, au fondement même de la connaissance animale, que le réel peut être décodé et prendre sens. Le point de vue propre à l'animal, son monde propre, son Umwelt ${ }^{2}$, représente un univers partiel orienté vers certaines finalités et, plus fondamentalement, la sauvegarde et le développement de l'espèce. Cette lecture utilitaire est profondément ancrée dans les dispositions les plus radicales ou les plus originaires du vivant.

Debuyst emprunte l'argument du « monde propre » à J. von Uexküll à partir de son célèbre exemple paradigmatique de cet insecte parasite qu'est la tique : «La tique a ceci de particulier que sa stratégie alimentaire consiste à guetter, perchée sur l'extrémité d'une brindille d'herbe, le passage d'animaux à sang chaud. Cet animal, privé d'yeux et sourd, perçoit l'approche de ses proies par son odorat. L'odeur de l'acide butyrique, que dégagent les follicules sébacées de tous les mammifères, agit sur lui comme un signal qui le fait quitter son poste et le lâcher en direction de sa proie » (Debuyst, 1985, p. 20). Cette présentation assez simplifiée de l'univers de la tique met ainsi l'accent sur cette sensibilité préalable fondamentale et ce mécanisme d'efficacité tourné vers la survie. L'Umwelt apparaît comme le monde central du vivant opérant un tri dans les signifiants, opération réglée
2 L'on ne s'étonnera guère que ce recours au concept d'Umwelt unifie, sur cet argument, problématiques éthologique et phénoménologique. Ce fondement préréflexif de la perception de l'environnement s'apparente à ce que Merleau-Ponty (1964, p. 17) appelle « foi perceptive ». Dans la tradition de la psychopathologie phénoménologique, I'on retiendra que ce sont les travaux de Binswanger qui ont systématisé l'articulation des notions d'Umwelt, de Mitwelt et d'Eigenwelt, que l'on peut traduire par « monde environnant», " monde interhumain " et «monde personnel » (Binswanger, 1963). 
phylogénétiquement. Si la richesse du monde extérieur disparaît, le rétrécissement utilitaire du milieu propre conditionne la sureté de l'action.

On pourrait ici bien évidemment discuter, comme d'autres l'ont fait, du passage d'une éthologie animale à une éthologie humaine. Il nous parait plus intéressant, dans le cadre de notre propos, de repérer que, pour Debuyst, cette référence permet de dégager un mode de fonctionnement premier et une structure fondamentale de la connaissance. À ce niveau de fonctionnement, la connaissance est prédéterminée par l'instinct, envisagé comme un ensemble de réactions préprogrammées selon certains schémas de comportements. C'est le monde propre qui ne retient de l'univers et dans cet univers que les éléments qui lui sont signifiants. Cette sensibilité préalable met en forme et organise une situation ordonnant des comportements tels que s'approcher ou s'éloigner, prendre ou lâcher, attaquer ou se défendre.

Bien que le passage vers l'éthologie humaine ne soit pas véritablement discuté dans l'ouvrage, il semble se dessiner en filigrane à travers la référence à la notion d'instinct humain empruntée à De Greeff. Cette aire d'influence donne ainsi l'occasion à Debuyst de marquer un pas de plus en insistant sur des dimensions supplémentaires faisant partie de la complexité d'une démarche active de prise de connaissance de l'homme par l'homme. La connaissance est ici affaire de processus psychiques, renvoyant à la psychologie même du « connaisseur » et pas uniquement à travers les limites de ses fonctions cognitives. L'orientation des processus de connaissance est radicalement marquée, déterminée même, par l'affectivité et la vie émotionnelle.

\subsection{L'animal avant Wittgenstein : modèle éthologique, jeux de langage et criminologie clinique}

Dans un récent ouvrage consacré à Wittgenstein, Debuyst (2014) propose une reprise des travaux de 1985 et de son épistémologie clinique mais à partir d'une autre perspective, grâce au concept de « jeu de langage » emprunté au philosophe. Le texte fait mention de l'animal dans deux chapitres, l'un visant la sensation et le jugement perceptif comme modes de codage de la réalité pour l'animal ; l'autre développant le 
fonctionnement de la pensée chez l'animal et chez l'homme dans leur manière d'agir. Ces deux chapitres, précédés de la définition du « jeu de langage », constituent, de manière équivalente à la thèse de 1985, une base conceptuelle sur laquelle l'épaisseur de la référence à Wittgenstein va reposer. Nous résumerons fortement le propos en deux points évoquant les liens entre langage et sensation ainsi que le passage de la tique à la grenouille.

\subsubsection{Le langage de la sensation}

L'auteur emprunte la voie des commentaires que fait Bouveresse (1995, 2004) de l'œuvre de Wittgenstein et de ses travaux sur Langage, perception et réalité. Comme en 1985, c'est la connaissance d'arrière-fond qui intéresse Debuyst, plus particulièrement la façon dont s'organise le rapport sensation/ perception en tant qu'il ne consiste pas seulement en une reproduction de la réalité. Ainsi, selon lui, ce rapport est déjà déterminé par une manière de voir cette réalité, de la reconstruire en fonction d'éléments liés au milieu ambiant ou à des impératifs plus nettement constitutionnels. En ce sens, il nous paraît évoquer une infrastructure du langage comme «sensation » permettant de le distinguer de la «perception ». Il souligne que ces "sensations sont canalisées dans un sens déterminé $e^{3}$ et qu'une série d'entre elles sont éliminées par incapacité d'être signifiantes dans le cadre de la construction perceptive » (Debuyst, 2014, p. 52).

Au fil des développements, deux déplacements s'opèrent entre 1985 et 2014, lesquels nous semblent résider, d'une part, dans la reconsidération de la sensation préétablie en tant que phénomène inné ; d'autre part, dans l'énoncé des implications cliniques concrètes et extensives que le dispositif d'écoute implique. En effet, Debuyst montre d'abord que la sensation est constituée comme un langage acquis, préréflexif, au même titre que la pensée, tout en précisant certaines nuances essentielles ${ }^{4}$. Dès lors, tant le langage lui-même que la sensation obéissent à la logique du codage. La thèse épistémologique se fait ici plus constructiviste encore qu'elle ne l'était. Ensuite, Debuyst affirme que l'écoute ne peut se réduire à son objet mais implique aussi le corps comme postures, mimiques, gestes... autant d'éléments non-verbaux - «éthologiques » pourrions-nous dire - à prendre en considération.
3 Souligné par l'auteur.

4 Voyez Debuyst (2014, pp. 55-57). 


\subsubsection{De la tique à la grenouille}

Si la tique a servi de premier exemple paradigmatique, c'est au tour de la grenouille de se rendre utile à Debuyst dans la mesure où il va reconsidérer le rapport sensation/perception en montrant que l'œil très élaboré du batracien réagit comme s'il y avait déjà perception au sens d'un jugement déterminant la réaction adéquate. De cette manière, l'antécédence de la sensation est contestée et la perception apparaît première et fondamentale. L'auteur reliera d'ailleurs la grenouille à la tique pour souligner que la recherche de sécurité pour l'animal ne rime pas avec l'ouverture de son monde à la complexité externe. Debuyst conclut cette séquence en réaffirmant, avec l'appui de Wittgenstein, que le langage n'est que l'extension de notre comportement animal. Enfin, il rapproche le philosophe de son maître De Greeff en établissant un air de famille entre « forme de vie », que l'on doit au premier, et «structure affective », que l'on doit au second.

Nous pensons que ce texte original nous permet à la fois de penser une phénoménologie des organes des sens - dans leurs différences essentielles - et une phénoménologie de l'écoute en tant qu'activité structuralement liée par enchaînements à l'ouïr, l'entendre et le comprendre. Avant d'écouter, il s'agit peut-être d'ouïr, autrement dit de reconnaitre que la langue est avant tout un système sonore avant de se signifier dans des formes plus complexes.

\subsection{De la tique à l'éthique}

L'analyse du fonctionnement de la pensée chez l'animal et chez l'homme débute par un retour sur « l'agir animal » et sur le problème singulier de la compréhension au double sens d'une connaissance non mécanique et d'une mécanisation de la connaissance. Debuyst revient alors sur la distinction entre l'animal et l'homme en faisant écho à ce qu'il avait déjà développé en 1985, à savoir l'utilité du comportement, défini au sens d'une action en cours et à réussir. Il existe des « proto-pensées $»^{5}$ tant chez l'homme que chez l'animal, devant

5 Terme que Debuyst emprunte à Dummett (1991). être considérées comme la « seule espèce de pensée qui est indispensable pour transformer les impressions sensibles en une perception » (Bouveresse, cité par Debuyst, 2014, p. 71). 
Dans une certaine mesure, en deçà de la pensée, l'intentionnalité spécifique de l'agir demeure à découvrir alors qu'elle se voit trop vite recouverte par le langage dans sa fonction transformatrice.

Debuyst établit alors un rapport d'équivalence entre l'agir animal et l'agir délinquant, au sens où l'animal et l'homme délinquant pourront «penser », en d'autres termes, prendre une distance par rapport à l'acte en y renonçant. Mais le propre de l'homme, "c'est qu'une pluralité de mobiles est susceptible de rendre compte d'un même acte, et que pourrait apparaître la prise de conscience de l'interdiction légale de l'acte et des conséquences qui peuvent en découler » (Debuyst, 2014, p. 73). Â partir d'un fait-divers donnant lieu à une étude de $\operatorname{cas}^{6}$, l'auteur nous apparaît ainsi inaugurer l'univers de l'éthique et de l'ethos animal en elle, tant pour la lecture du passage à l'acte que ce qu'elle requiert comme compétences pour le criminologue clinicien. Malgré des intentionnalités apparentes dans certaines pensées, il nous faudra toujours nous demander si nous ne sommes pas en présence de proto-pensées, comme univers emprisonnant, sans alternative, se déroulant uniquement dans le cadre de la pure réalisation matérielle de l'acte, tout autre événement-obstacle apparaissant fortuit et inattendu ${ }^{7}$. Et si le langage joue, c'est peut-être simplement pour préciser la manière la plus adéquate de réussir l'action elle-même.

Enfin, l'idée d'une «pathologie du langage », au sens d'un jeu de langage profondément réducteur, "fermé et enfermant », arrive à point nommé pour traduire cette restriction des possibles et des alternatives opérant dans une connaissance délinquante - et, en miroir, une connaissance de la délinquance si elle ne reste pas ouverte. Debuyst renoue ici avec les enseignements de De Greeff ${ }^{8}$, surtout lorsqu'il montre la logique implacable de l'instinct de défense qui ne serait plus dialectisé, aboutissant à une pure expression de la destruction. Aucun autre jeu de langage ne pourrait venir y introduire du « jeu » au sens d'un espace ou, comme le dit Debuyst, de « biais pour qu'un tel jeu puisse se modifier en révélant ses failles et donner une place à un autre type de préoccupation [...]» (Debuyst, 2014, p. 80).
6 Voyez Debuyst (2014, pp. 74-77).

7 On sent ici toute l'influence de la pensée de De Greeff lorsqu'il traite des instincts et de la restriction des possibles que représente l'instinct de défense. Toutefois, la "désintentionnalisation" opérée par le jeu de l'instinct de sympathie, aboutissant également à intégrer l'idée de hasard comme capacité à se distancier d'une reconstruction de l'autre comme figure menaçante ou visant intentionnellement à nuire, nous paraît créer une tension entre Debuyst et son maître. En effet, le statut théorique de l'intentionnalité serait à étudier de plus près chez les deux.

8 On peut lire entre les lignes la référence à la théorie de la criminogenèse et à la psychologie de l'assassinat, plus particulièrement quand De Greeff évoque cette situation où l'auteur d'un homicide va fréquenter des groupes sociaux ou d'appartenance qui vont dévaloriser la victime pour le renforcer dans son projet criminel. On pourrait dire que l'auteur cherche à confirmer son jeu de langage et à s'écarter de tout ce qui pourrait le conflictualiser. 
9 Albert Demaret (19332011) était psychiatre et prenait plaisir à se présenter, d'abord et avant tout, en tant que naturaliste et éthologue. II exercera à Liège en milieu hospitalier durant toute sa carrière et fut un pionnier à la reconnaissance internationale de l'approche évolutionniste et éthologique en psychopathologie, notamment grâce à la publication de son livre Éthologie et psychiatrie (1979) qui a été réédité et augmenté récemment (2014).

10 Par commodité et esprit de synthèse, nous n'indiquerons plus systématiquement les références à Éthologie et psychiatrie et ne ferons pas l'exercice qui consisterait à présenter les autres publications dans lesquelles Demaret évoque également

- et parfois peaufine

- ses travaux. Une présentation exhaustive de ses travaux avec la bibliographie complète peut être consultée dans Englebert et Gauthier (2011) et dans la nouvelle édition de son livre (2014).

Parmi les articles ultérieurs à Éthologie et psychiatrie, citons tout de même La psychiatrie évolutionniste (1991) et Le divan naturel (1994).

11 Demaret s'amusait d'ailleurs à rappeler que " si nous ne sommes pas des bêtes... les animaux non plus! ».

\section{2. Éthologie et psychopathologie : l'apport d'Albert Demaret}

L'essence du travail d'Albert Demaret' ${ }^{9}$ se trouve dans son magnum opus - et unique ouvrage - Éthologie et psychiatrie $^{10}$. L'adjectif est probablement galvaudé à force d'être trop usité, mais voici là, véritablement, un ouvrage révolutionnaire. Publié en 1979, l'auteur y propose une synthèse de ses travaux consacrés à la compréhension de l'homme affecté d'un trouble psychiatrique, dans une perspective éthologique et évolutionniste. Demaret s'efforce de mettre en lumière la dimension adaptative et fonctionnelle des sémiologies psychiatriques, tout en observant les analogies entre le comportement humain et celui des animaux ${ }^{11}$. La consécration la plus prestigieuse à propos de son travail est probablement le témoignage que lui rendent les grands représentants de la psychiatrie évolutionniste (Price et al, 2007) ${ }^{12}$ en retraçant l'évolution de la réflexion sur le territoire en psychopathologie, en prenant comme temps zéro de l'histoire de ce concept les propositions de Demaret sur la psychose maniaco-dépressive. Précisons que, si Debuyst ne traite au fond qu'indirectement - mais sérieusement - d'éthologie, Demaret donne toute son attention à cette discipline (que l'éthologie soit animale ou humaine). À l'inverse, si Debuyst s'inscrit de plainpied dans le champ de la criminologie clinique, Demaret n'évoque cette matière qu'en la frôlant. On remarquera donc cet étonnant chiasme qui se révèle fondateur pour notre proposition.

La section de cet article dévolue à présenter les travaux de Demaret - dont la grande originalité a peut-être paradoxalement contribué à les rendre trop méconnus - est partagée en trois points. Nous présentons d'abord - de façon trop brève - le contexte épistémologique de ses propositions (2.1.). Ensuite, nous synthétisons trois modèles psychopathologiques représentatifs de son œuvre - l'anorexie mentale, la psychose maniaco-dépressive et l'hystérie (2.2.). Enfin, nous évoquons une timide mais géniale incursion dans le champ de la problématique criminologique (2.3.). 


\subsection{Psychopathologie, éthologie et psychiatrie évolutionniste}

Demaret cherche à fonder une réflexion sur une psychopathologie générale plus ou moins commune à l'homme et aux animaux. Cette double approche clinique et éthologique est basée sur l'observation ; celle du monde animal et celle de ses patients. Celles-ci lui permettent d'identifier les dimensions adaptatives de nombreux troubles psychopathologiques comme la paranoïa, l'anorexie mentale, l'hystérie, la schizophrénie, le trouble obsessionnel compulsif, etc.

Parallèlement à cette utilisation des observations du monde animal et des analogies qu'il constate avec le comportement humain, les théories évolutionnistes influencent également sa réflexion. Celles-ci reposent sur un second paradigme, celui de la phylogenèse. En effet, aux côtés des analogies hommeanimal, viennent se greffer des analogies homme-homme ancestral $^{13}$. Dans cette considération phylogénétique, les notions de milieu naturel et d'environnement occupent une place primordiale. Nous ne sommes plus dans une perspective où le comportement est prioritairement observé, mais plutôt recontextualisé selon une variation de l'espace et/ou du temps.

Si la psychiatrie évolutionniste dans laquelle s'inscrit Demaret prend en considération le caractère génétique et héréditaire des sémiologies psychiatriques, elle refuse cependant de les envisager sous le seul angle de la pathologie et du trouble. C'est à partir de ce postulat que Demaret se livre, sur base de sa pratique clinique, à l'examen systématique de nombreux troubles psychopathologiques pour en identifier les dimensions adaptatives. Demaret suggère ainsi que nombre de comportements humains, considérés comme pathologiques à ce jour, devaient avoir une valeur adaptative dans le milieu originel qui a façonné la morphologie et le psychisme de notre espèce. Une variation du temps - un comportement à une autre époque - ou de l'espace - un comportement dans un autre contexte, une autre situation sociale, culturelle, économique - peut conférer à tout symptôme psychopathologique une dimension adaptative et fonctionnelle. Il n'est guère question ici de chercher une signification ou un causalisme psychiques reliés à l'acte du sujet. Par contre, grâce au « jeu » des analogies, l'on peut faire ressortir les racines phylogénétiques
12 Une synthèse en français de ce texte référence pour la psychiatrie évolutionniste a été récemment publiée. Voyez Price (2016).

13 Nous n'insisterons pas sur l'importante nuance qu'il y a à faire entre les perspectives éthologique (hommeanimal) et évolutionniste (homme-homme ancestral) et sur les nombreuses critiques qu'il y a à formuler à l'encontre des psychologie et psychiatrie évolutionnistes. Sur ces questions, nous renvoyons à Englebert et Follet (2014).

Précisons toutefois que les travaux de Demaret s'inscrivent plus résolument dans une perspective éthologique, définie comme l'observation du comportement (animal, et donc humain) en milieu naturel. 
14 Précisons que, dans une publication récente, le Professeur Stanley Coren (University of British Columbia) révèle que Freud était souvent accompagné de son fidèle chien, Jofi, durant les séances d'analyse. Son fidèle compagnon qui, à l'origine, était dans la pièce pour réconforter le psychanalyste, se serait imposé comme un élément déterminant pour les patients. Freud observait que ces derniers étaient plus détendus et que l'animal semblait aider les patients pendant leurs séances de thérapie à parler plus ouvertement. Dans diverses notes théoriques, Freud prônera l'utilisation d'un chien durant la thérapie en raison du caractère neutre que celui-ci pouvait renvoyer au patient, qui semblait interpréter cette étrange présence de façon apaisante (Coren, 2010, pp. xvi-xvii). adaptatives de certains symptômes psychiatriques en recadrant ceux-ci dans un autre espace-temps.

Demaret rappelait souvent que Freud avait été incompris lorsqu'à plusieurs reprises, il confiait son intuition d'une proximité entre l'ontogenèse et la phylogenèse. À la fin de sa vie, ce dernier écrivait : «Avec les névrosés, on se croirait dans un paysage préhistorique, par exemple dans le Jurassique. Les grands sauriens sont toujours là, les joncs et les prèles sont aussi hauts que des palmiers » (Freud, 1941, cité par Demaret, 1979, p. 15) ${ }^{14}$. Ainsi, celui qui a révélé 1 'importance de la vie infantile dans la formation des névroses et de la personnalité (l'ontogenèse), attire également l'attention sur celle du passé de l'espèce (la phylogenèse). Le symptôme, dont l'intérêt a pu être négligé dans la psychopathologie d'orientation psychanalytique au profit de l'analyse des conflits sous-jacents, fait l'objet d'une attention renouvelée dans l'œuvre de Demaret. En lui reconnaissant une composante phylogénétique possible, on lui découvre en même temps un sens fonctionnel nouveau, utile à la compréhension de la nature et de l'origine des troubles présentés.

\subsection{Trois grands modèles psychopathologiques}

\subsubsection{Anorexie et altruisme}

Le symptôme le plus visible et le plus préoccupant de l'anorexie mentale est le refus de s'alimenter, sans cause organique, pouvant conduire un état d'amaigrissement très important voire, dans les cas les plus extrêmes, à la mort. En parallèle à ce refus alimentaire, Demaret estime essentiel de s'intéresser aux signes considérés comme secondaires, mais qui sont classiquement associés au refus de manger. Il relève la nette prédisposition pour le sexe féminin, les crises de boulimie, les vomissements, l'aménorrhée (qui peut être la conséquence de la sous-alimentation mais survient parfois précocement), l'absence habituelle de dépression et la négation de l'état de maigreur, l'anosognosie, la conservation d'une grande activité physique et mentale et l'altruisme alimentaire.

La première dimension paradoxale du trouble est que l'anorexique présente un intérêt profond pour la nourriture : elle a généralement une grande connaissance de la diététique, 
cherche à nourrir autrui et cuisiner pour son entourage, vole des aliments pour les dissimuler dans des cachettes, semble souvent obsédée par la nourriture et, rappelons-le, peut présenter des accès de suralimentation. La seconde dimension paradoxale du trouble anorexique est l'hyperactivité caractéristique de ces jeunes filles d'apparence si faible et fragile, qui assument souvent une activité physique et intellectuelle impressionnante. Bref, lorsque la maladie ne prend pas ces proportions extrêmes, les anorexiques possèdent d'étonnantes capacités adaptatives à leur milieu.

Un comportement qui retient particulièrement l'attention de Demaret est l'altruisme alimentaire, qui peut être considéré comme " la composante la plus fondamentale du syndrome [alors que] la perspective médicale mettant l'accent sur [le refus alimentaire et la perte de poids qui en découle] empêche de reconnaître d'emblée la valeur adaptative du syndrome entier » (Demaret, 1979, p. 152, mis en italique par nos soins). Pour comprendre la dimension fonctionnelle de cette tendance à nourrir l'autre, il faut se souvenir que la valeur adaptative d'un comportement n'est pas forcément orientée vers l'individu, mais s'étend au groupe auquel il est apparenté. De ce point de vue, que dire d'un individu qui consomme très peu de nourriture, reste actif et résistant tout en cherchant à nourrir les autres membres du groupe ? Dans des conditions extrêmes (par exemple de famine), cet avantage est particulièrement déterminant pour les enfants du groupe qui nécessitent une grande attention et sont dépendants des autres pour se nourrir. Demaret rappelle d'ailleurs que les vomissements ou régurgitations sont des modes de nourrissage très répandus dans le monde animal (et dans plusieurs sociétés humaines).

Parler de l'intérêt pour les enfants nous amène à une troisième dimension paradoxale de l'anorexie. Alors qu'elles sont biologiquement incapables de procréer (aménorrhée), les anorexiques présentent un grand intérêt pour les enfants. Elles sont baby-sitters, responsables de mouvement de jeunesse, puéricultrices, infirmières pédiatriques, logopèdes, éducatrices, institutrices, etc. Cette observation permet de généraliser la notion d'altruisme, qui ne se réduit pas à la sphère alimentaire.

Demaret trouve, à travers le comportement de femelles primates s'occupant du nouveau-né de leur mère ou de leur 
sœur, un modèle animal correspondant à cet altruisme anorexique. Ces femelles, qui n'ont pas d'enfant, vont éduquer et se préoccuper d'un enfant qui n'est pas le leur mais qui fait partie de leur environnement proche. Elles endossent la fonction de tuteur et jouent un rôle dans l'épanouissement social des jeunes. En outre, elles nourrissent ces nouveau-nés, pouvant se priver pour le bien-être de la nouvelle génération. Demaret relève également la fonction de perpétuation (profitable pour le groupe) qu'induisent qu'induisent le déni et la dysmorphophobie. Ces perspectives sont révolutionnaires en termes de prise en charge thérapeutique et suggèrent de manière innovante de moins se focaliser sur la perte de poids (sans la nier) et d'explorer ces autres signes typiques généralement considérés comme accessoires. De ce point de vue, les thérapies comportementales basées sur l'observance du gain de poids avec à la clé un système de récompenses et de punitions se révèlent tout simplement obsolètes.

\subsubsection{Manie, mélancolie et territoire}

En observant ses patients maniaco-dépressifs, d'une part, et les animaux dans la nature, d'autre part, Demaret constate que « rien ne ressemble autant à l'agitation d'un maniaque que celle d'un animal territorial » (Demaret, 1979, p. 115). Lorsqu'il est sur son territoire, l'animal semble posséder un « avantage psychologique » sur autrui, « comme si ses forces étaient décuplées » (Ibid.). L'espace familier confère à l'autochtone un avantage psychologique et physique sur l'envahisseur. Sur son territoire, les caractéristiques essentielles de l'animal sont l'agressivité, un plus grand succès lors de l'affrontement même face à des adversaires de taille plus imposante, et des comportements de séduction face aux femelles. Les limites de l'espace sont marquées par des cris, des signaux visuels (coloration des organes sexuels ou d'une partie du corps) et olfactifs (dépôts d'urines ou d'excréments). À l'échelle humaine, ces comportements sont superposables à ceux du maniaque : d'une grande assurance et estime personnelle, il est agressif et ironique et, fort d'un sentiment de toute-puissance, défie socialement son vis-à-vis quel que soit le statut de celui-ci. Hyperactif et euphorique, il est à la recherche de sensations nouvelles et extrêmes. Il est bruyant, excentrique, et sa vie sexuelle est débridée. 
Le fonctionnement maniaque étant susceptible de se prolonger dans le temps - rappelons que, sans intervention pharmacologique, l'état maniaque peut perdurer plusieurs mois -, une telle hyperréactivité, sans période de repos ou presque, représente selon les circonstances une réelle adaptation. L'on peut d'ailleurs se demander si notre société ne prend pas l'état maniaque pour idéal à travers l'image du jeune cadre dynamique hyperactif. Ces jeunes hommes, brassant des sommes inconcevables d'argent, qui semblent ne vivre que pour leur travail, s'y trouver jour et nuit, quasiment sans dormir, sortant jusque tard dans la nuit tout en étant opérationnels le lendemain à la première heure, correspondent, en de nombreux traits, à la symptomatologie maniaque. Certains hommes politiques ou hommes d'affaires en sont les parangons célèbres.

Ce jeu des analogies entre le comportement de l'animal territorial et celui du maniaque laisse à penser que ce dernier « se comporte partout comme s'il était chez lui » (Ibid., p. 117). Nous allons voir que le comportement du mélancolique/dépressif, de façon diamétralement opposée, se rapproche plutôt de celui de l'animal qui s'aventure sur le territoire d'un congénère. Cet animal perd aussitôt toute combativité et toute séduction. Il fuit face au propriétaire du territoire, même si ce dernier apparaît plus faible. Devant une femelle, peureux, il ne se risque pas à une parade amoureuse. Alors que le maniaque serait partout comme chez lui, le mélancolique serait un animal « déterritorialisé ». Il se sent gênant, « de trop », presque fautif «d'être-là ». Figé ou fuyant, il est incapable d'affronter la « compétition » sociale et semble avoir perdu tout désir sexuel.

Cette analogie des attitudes maniaques et mélancoliques avec celles des oiseaux ou poissons territoriaux amène une nouvelle manière de voir les choses, déplace le regard du clinicien sur des comportements que la psychiatrie conventionnelle ne retient pas comme caractéristiques des psychopathologies. L'identification du rapport à la territorialité comme structure fondamentale permet de voir au-delà des signes classiquement attribués au trouble bipolaire comme la fluctuation de l'humeur. Ainsi, si ces modèles induisent un changement, celui-ci réside peut-être en priorité chez le clinicien car, une fois l'analogie révélée, il n'est souvent plus possible 
de concevoir le phénomène clinique sans avoir à l'esprit ces étonnantes considérations.

\subsubsection{La fonction du comportement hystérique}

La « feinte de l'aile brisée » (Demaret, 1979, pp. 94-96), bien connue des ornithologistes, est un comportement manifesté par certaines espèces d'oiseaux nichant sur le sol, qui consiste à feindre la fuite sans pour autant s'envoler, et survenant lorsqu'un prédateur s'approche du nid. Le prédateur entreprend dès lors de suivre l'oiseau «blessé » qui apparaît comme une proie facile, mais ce dernier ne cesse de s'éloigner à chaque fois que le prédateur approche jusqu'à finalement s'envoler lorsque ce dernier a été suffisamment éloigné du nid.

Demaret compare la feinte de l'aile brisée au comportement hystérique. Les comportements de provocation, de paralysie fonctionnelle, d'ambivalence, etc., passés sous le prisme de l'éthologue, permettent de mieux comprendre le mystère relationnel inhérent à l'hystérie, tout en situant le propos dans la mise en évidence d'une fonction. Aussi, que ce soit pour l'oiseau qui recourt à la feinte de l'aile brisée ou pour le comportement hystérique - par exemple de paralysie ou d'attraction de l'attention vers soi, et tout ce que l'on appelle les bénéfices secondaires -, la fonctionnalité protectrice de tels comportements se révèle. L'intentionnalité hystérique révèle sa fonction à travers les analogies avec l'intentionnalité des pluviers pratiquant la feinte de l'aile brisée. Cette «optique» pousse alors le clinicien à se demander quel pourrait être l'objet de la protection histrionique de l'hystérique.

\subsection{Une timide incursion (intuition de génie) dans le champ de la criminologie}

Si l'apport de Demaret à la compréhension de l'homme affecté d'une psychopathologie est évident, il n'a, à l'inverse de Christian Debuyst, jamais directement dialogué avec les matières criminologiques. Toutefois, une intuition géniale, qu'il ne développe pas, apparait comme presque perdue dans son livre. Cette proposition, d'apparence interpelante voire provoquante, repose sur une remarquable évidence : « ... La fonction sociale des psychopathes dépend des conditions du 
milieu. Plus celles-ci sont perturbées, plus le "déséquilibré" se révèle adapté et même utile. En temps de paix, on les enferme ; en temps de guerre, on compte sur eux et on les couvre de décorations...» $(1979$, p. 29). Cette remarque, qu'il formule de façon presque détachée, n'est pas dénuée d'importantes conséquences. Elles nous conduisent à considérer la psychopathie - qui, selon nous, ne doit pas être unilatéralement reliée aux matières criminologiques - comme un fonctionnement psychologique que l'on n'arrivera pas à comprendre correctement si l'on se borne à le considérer comme un déficit ou à lui identifier des déficiences.

À partir de cette proposition de l'adaptation du psychopathe en certaines circonstances, nous pensons que l'incompétence émotionnelle qui lui est classiquement attribuée - et tout particulièrement le trouble généralisé de l'empathie - doit être entièrement repensée. Si l'empathie est la faculté à de comprendre émotionnellement l'autre et la possibilité de comprendre les choses de son point de vue, il est sans doute plus raisonnable de penser que le psychopathe est un être particulièrement compétent en la matière. C'est précisément ce qui en fait un être redoutable, car sa compétence émotionnelle - avantage adaptatif social maximal - le rend infaillible et explique la maitrise et l'emprise qu'il peut exercer sur autrui. Dès lors, c'est bien d'un trouble de la sympathie que souffre le psychopathe - allant de pair avec sa froideur émotionnelle -, mais certainement pas, comme l'annoncent la majorité des conceptions modernes de ce diagnostic, un trouble de l'empathie ${ }^{15}$.

\section{Ethos et adaptation : le gain d'intelligibilité pour la criminologie clinique}

La référence à l'éthologie est sans nul doute un réservoir riche de propositions fécondes tant du point de vue d'une épistémologie clinique de la criminologie que d'une psychopathologie soucieuse de saisir le monde propre de la maladie vécue. Ici, nous entendons nouer un dialogue plus serré entre Debuyst et Demaret en insistant sur les ponts qui nous paraissent pouvoir être jetés entre ces deux œuvres qui, étonnamment, ne se sont jamais rencontrées frontalement, tout en prolongeant ponctuellement leurs thèses. Cette lecture croisée s'articule
15 Nous nous permettons de renvoyer, concernant la dimension adaptative de la psychopathie, à Adam (2015) et Englebert $(2015,2016)$ et, sur les apories inhérentes au diagnostic de personnalité antisociale, à Englebert et Adam (2016). 
en trois points. Premièrement, tout en montrant en quoi ces deux auteurs nous aident à mieux penser une critique d'une lecture exclusivement négative du fait pathologique comme du fait délinquant, nous mettrons en évidence l'importance décisive d'une normativité subjective, en vue d'éviter de la confondre avec d'autres ordres normatifs comme le jugement de valeur ou la moyenne statistique s'imposant souvent dans les lectures actuelles, lesquelles entendent démontrer une inadaptation. Deuxièmement, à partir du langage et de sa valence adaptative, nous serons amenés à considérer son infrastructure en contribuant aux analyses de la délinquance comme jeu de langage ou de la pathologie comme langage, avec une proposition qui s'ancre dans l'anthropologie médicale philosophique. Enfin, en toute inspiration phénoménologique, nous reviendrons à la racine de l'expérience et de l'épreuve cliniques, à travers les activités de l'oü̈r et du voir, signes de notre animalité, ce qui nous permettra de suspendre les prétentions trop fréquentes d'une criminologie clinique parfois exagérément préoccupées par la production d'une signification, au risque de laisser notre animalité et nos organes des sens dans le silence inouï et invraisemblable du raisonnement.

\subsection{Au-delà d'une psychopathologie des failles : à la recherche d'une normativité subjective}

Dans le champ de la psychopathologie, Demaret propose, tout en conservant la primeur accordée à l'observation, une subtile variation de méthode par rapport aux chefs de file que sont Harlow et Bowlby. Là où ces derniers cherchent à comprendre un comportement humain fondamental et la possibilité de son absence - marquant l'apparition du trouble -, Demaret ne s'inscrit pas dans cette « logique du manque » (Englebert et Follet, 2014). Ses travaux ne cherchent pas à objectiver un « pathologique » qui fonctionnerait telle une dérivation du « normal ». Dans cette perspective que ses travaux renient, le trouble est compris comme la dysfonction d'un comportement par rapport à une norme. Dans la psychopathologie telle que nous la révèle Demaret, l'on cherche plutôt la logique du fonctionnement pathologique. Le trouble devient l'expression d'un fonctionnement qui se révèle inadapté dans certaines conditions, adaptatif dans d'autres. Cette démarcation signée 
par Demaret s'avère bien plus qu' anecdotique. Cette façon de penser la psychopathologie correspond en fait au millimètre près à la suggestion de Minkowski (1966) qui prônait une «psychologie du pathologique » et encourageait à renoncer à la construction chimérique d'une hypothétique «pathologie du psychologique ». Considérer que le psychologique exempt de tout pathologique puisse exister et servir d'objet d'étude, induit selon le psychiatre phénoménologue une référence implicite à « une norme abstraite à peine viable » (Ibid., p. 64) et un modèle illusoire qui représenterait « des opérations de l'esprit bien plus que la réalité vivante » (Ibid.).

Les travaux de Demaret luttent, selon nous, contre une clinique « consignée dans les manuels, soigneusement épurés souvent de tout ce qu'il y a de vraiment humain dans notre existence » (Ibid., p. 65). Cette volonté apparemment implicite de Demaret, qui consiste à ne pas réduire le pathologique à une dérivation de la norme, correspond également parfaitement aux thèses développées par Canguilhem dans Le normal et le pathologique (1966). La thèse principale de ce célèbre ouvrage est de considérer le pathologique comme une expérience innovante et « positive », qui, plus que de s'écarter de la norme existante, crée une normativité inédite. Le «normal » et le «pathologique » ne peuvent être déterminés de manière absolue, ils ne se révèlent qu'en situation ${ }^{16}$, dans la relation concrète qu'un individu entretient avec son milieu : «Le vivant et le milieu ne sont pas normaux pris séparément, mais c'est leur relation qui les rend tels l'un et l'autre » (Ibid., p. 90). L'adaptation doit donc être considérée comme la capacité de changer de milieu sans se mettre en danger et d'envisager, dans un certain équilibre, une modification autant de soi-même que de l'environnement.

Dans le domaine de la criminologie clinique, Debuyst a toujours vivement critiqué ce qu'il appelait une psychologie ou une psychopathologie des failles, témoignant avant tout d'une défaillance de contextualisation. C'est alors plutôt la psychopathologie qui est défaillante, bien avant qu'elle se propose d'objectiver la pathologie comme défaut. Dans cette veine, la déconstruction du concept de "personnalité criminelle » constitue d'ailleurs un moment critique important dans l'histoire des savoirs sur le crime et la peine, qui montre à quel point les interprétations positivistes du fait
16 Pour une réflexion plus approfondie sur les travaux de Canguilhem et leurs apports à la psychopathologie, voyez Englebert (2013) et Adam (2015). 
17 Ladite renvoie à la position par défaut concernant un phénomène donné. délinquant l'infléchissent déjà de toute une caractérisation, le plus souvent extérieure à l'environnement dans lequel le fait est survenu. Debuyst le met en exergue de manière particulièrement habile, dans un article qui a fait date, où il démontre en quoi un examen attentif des données atteste du « caractère uniquement négatif des appréciations [...]» (Debuyst, 1977, p. 223). Parmi d'autres arguments, relevons que le traitement de la variable «impulsivité », trait a priori stable de la personnalité criminelle et de la psychopathie, nous donne la possibilité de voir qu'elle peut aussi participer à sauver des vies dans certains contextes. La prise en compte du contexte change la donne, et nous voyons bien comment cette proposition critique fait écho à l'intuition de Demaret. Debuyst aura d'ailleurs cette formule dont on retrouve des accointances évidentes avec la proposition du psychiatre liégeois : «On pourrait évidemment dire qu'en temps de guerre, les sujets dont la personnalité présente des nuances psychopathiques sont rois » (Ibid., p. 224).

Nos deux auteurs nous obligent, au sens d'une exigence clinique, à poursuivre la critique de ces perspectives aveuglées par les manques, les défauts, les défaillances, les inadaptations... tout un cortège de notions qui témoignent certainement d'un souci d'inscrire la négativité dans la compréhension des phénomènes psychiques. Le problème est que cette négativité constitue souvent l'unique prisme à travers lequel le pathologique est vu. Qu'il suffise pour s'en convaincre de lire la plupart des expertises psychodiagnostiques insistant exclusivement sur ce que l'homme n'est pas, dans une logique de $l^{\prime}$ " hypothèse nulle ${ }^{17}$, et de ses déficits jugés de façon normative. Cette perspective compréhensive néglige savamment ce qu'est l'homme, duquel on réduit à peau de chagrin toute forme de perspective dialectique. Au-delà de l'empirie de ces expertises de la faille, les classifications psychiatriques nous révèlent le plus souvent une logique d'inadaptation sous-jacente à la présentation raisonnée d'un trouble. Qu'une lecture experte, supposée objectivante, ne puisse pas avoir accès à un univers étrange et étranger, inobjectivable dans le langage opératoire des concepts qu'elle se donne, telle est une réalité qui ne se laisse pas si aisément reconnaître. Or, l'ancrage phénoménologique nous amène nécessairement à approcher un monde vécu dans sa positivité et non comme soustraction d'un monde normé pour le plus grand nombre. 
Notre proposition d'une «normativité subjective », au sens où elle s'observe du point de vue du sujet lui-même, devrait, selon nous, guider les interventions cliniques et s'inscrire comme un paradigme méthodologique central de la criminologie clinique de demain. Cette notion de subjectivité indique, par exemple, les notions de bien-être propres à chacun ou le fait de se sentir heureux, de se trouver en bonne forme corporelle, etc. Ces états peuvent être vécus comme des désirs de correspondre à un idéal de valeurs ou d'être comme ce que sont et ce que font le plus grand nombre de gens. On pourrait parler d'un désir de conformité. Au contraire, certains peuvent avoir un désir d'originalité, de non-conformisme ou d'anticonformisme. Nous sommes alors face à l'idéal auquel le sujet aspire. L'on peut se référer ici à la figure de l'athlète qui ne se sent en accord avec un modèle ou un idéal valorisé qu'en étant sans cesse en train de dépasser les normes moyennes des autres et les siennes. Cette figure idéale est particulièrement agissante dans nos sociétés obsédées par la performance. Dans ce cas, cette dynamique tournée vers l'expansion de soi, au-delà de ce qui a été acquis, est productrice de nouvelles normes et valeurs. Or, la normalité statistique est insuffisante pour rendre compte de cette normativité dynamique, inhérente à la vie elle-même, en quête de nouveaux équilibres.

Ce type de normativité est donc résolument dynamique. Il contraste avec les normes que l'on établit par convention avec les prétentions d'une certaine stabilité durable. La normativité est ici mouvante, en constante instance de dépassement possible. Nous sommes dans l'ordre d'une normativité ou d'une normalité toujours en train de se mettre en œuvre, et renvoyant à des critères comme ceux de bien-être, de santé, de qualité de la vie. La question clinique à se poser devient : Est-ce que telle modalité de comportement ou telle forme d'existence, entrave ou non la capacité de jouir de la vie, de se plaire à exister, la capacité d'agir, de réaliser ses projets et désirs, de participer à l'entreprise de progrès et d'accomplissement, non seulement de soi-même mais aussi de la société, des autres, du monde, etc. ? L'on saisit intuitivement que ce registre de la norme subjective et dynamique ne peut pas se définir indépendamment du rapport au milieu. Ainsi, l'être humain est d'une certaine façon également normal ou anormal par rapport à lui-même. 
Ce constat nous oblige à adopter, à travers une nouvelle suture phénoménologique, une perspective historique ou temporelle, renvoyant essentiellement à la transformation de soi au cours du temps. Le point de vue du sujet est ici primordial car il se juge par rapport à lui-même. Ainsi, ce dernier peut se dire qu'il n'est plus dans son état normal ou dans sa forme habituelle, qu'il se trouve en situation critique parce qu'il n'est plus adapté à des circonstances actuelles et inédites. Comme il est appelé à évoluer, à se métamorphoser lui-même, il ne serait plus normal s'il restait le même. Il lui faut alors réaliser des possibilités propres supplémentaires qui n'ont pas encore été actualisées, en accord avec ces circonstances inédites. Autrement dit, il lui faut lui-même pouvoir inventer ou innover des formes d'être et de se comporter qui soient normatives, c'est-à-dire à la fois qui lui conviennent de son point de vue personnel et qui soient accordées en référence à son environnement lui-même changeant.

À bien y regarder, s'adapter à la moyenne ou à la normalité sociale, dans ces circonstances contraignant à la métamorphose de soi, pourrait s'avérer pathologique. On pourrait alors parler de «normopathies », soit les malades de la normalité. C'est ce que Canguilhem (1966, p. 205) appelle les «maladies de l'adaptation » ou ce qu'un auteur comme Sami-Ali (1980) a étudié à travers le vocal de «banal ». D’un point de vue dynamique, l'homme «normal » est «normatif », capable de ne jamais cesser de se normer, de se réguler, ce qui implique qu'il puisse donc se créer de nouvelles formes de vie qui soient adaptées à ce que sont ses conditions de vie. Il s'agit aussi de pouvoir rencontrer les transformations auxquelles l'appelle son devenir d'âge en âge. Tandis qu'à l'inverse, une anormalité peut alors correspondre à une fixation plus ou moins rigide à un ensemble de degrés de réaction. En d'autres termes, une anormalité peut correspondre à une incapacité de répondre dans des situations nouvelles et donc de trouver comment pouvoir s'y adapter et s'y comporter d'une façon adéquate. Il existe des circonstances, des événements, des changements où il ne serait pas normal de se conduire à la manière de l'homme moyen, normal au sens statistique, ni à la manière la plus habituelle qu'est la nôtre ou qui serait celle du plus grand nombre. Nous suggérons donc de considérer que l'homme normal n'est pas nécessairement quelqu'un, en 
toutes circonstances, identique et égal à lui-même. Le même toujours et partout, pareil, soit à son modèle le plus habituel, soit à la moyenne, celle du commun des mortels.

Il découle de ce raisonnement une conception doublement relative des normes : d'une part, une relativité individuelle et subjective et, d'autre part, une relativité liée aux circonstances et au milieu. Il n'est par conséquent pas possible de porter un jugement absolu, d'un point de vue extérieur, en déterminant une normalité en soi et une anormalité en soi, laquelle serait valable pour tous et partout et en toutes circonstances. Il n'est pas possible non plus de se référer à des moyennes objectives, telles que la science peut chercher à les établir.

\subsection{La pathologie comme langage : un retour pathique au verbe}

À côté de la critique des vertus inadaptatives des pathologies, nous pouvons donner une autre amplitude à la réflexion en portant notre attention sur l'idée de la délinquance et de la pathologie comme des formes d'adaptation radicale ${ }^{18}$. La référence récente de Debuyst à Wittgenstein - et plus largement à une psychopathologie fécondée par la philosophie ${ }^{19}$ - nous permet de proposer un saut logique : de la pathologie du langage à la pathologie comme langage. Par conséquent, nous pouvons considérer la connaissance délinquante comme un « jeu de langage pathologique sans jeu », au sens de cette nécessité au fonctionnement d'un mécanisme : il faut qu'il puisse y avoir $\mathrm{du}$ « jeu » pour qu'il fonctionne. Un jeu où le joueur serait le jouet du jeu, joué par le jeu... Ici, le langage psychopathique de l'action pour l'action elle-même, tel un carpe diem pulsionnel, semble alors éclairer le paradigme psychopathique en tant que langage s'inscrivant, comme le dit Kinable, dans la « circularité d'une activité sans fin(s) »(Kinable, 1998, p. 122). En ce sens, il importe de revenir sur les verbes avant leurs compléments d'objet tels qu'ils se donnent à entendre dans le langage de nos patients. Ce mouvement de retour vers le verbe prend tout son sens dans l'anthropologie médicale philosophique de von Weizsäcker qui identifie le "paysage pathique », défini comme "monde perçu sur un mode passionnel, dans la vie vécue et en même temps éprouvée [...] » (von Weizsäcker, 2011, p. 55). Il identifie à ce paysage cinq catégories qui sont
18 Au sens précis de la notion de racine, à la base même de l'existence.

19 Parmi d'autres, nous nous permettons de mentionner la journée d'étude « Le philosophe dans la psychopathologie » qui s'est tenue à l'Université de Liège le 2 octobre 2015, à laquelle les deux auteurs de l'article ont participé. 


\section{Pour proposer une dialectique supplémentaire à cette série, nous pourrions y inclure le verbe "savoir". \\ 21 L'on ne peut que renvoyer aux belles analyses de Kinable (1998) lorsqu'il se propose de saisir le verbe et ses inflexions dans la grammaire psychopathique.}

\section{On peut ici citer} deux exemples féconds : I'un tiré des travaux de J. Kinable (1990) lorsqu'il établit des rapports entre les processus de deuil et de passage à l'acte délinquant à partir de l'étymologie du terme " délinquant »; l'autre emprunté à Ricœur (2004) qui enrichit la conceptualisation de la notion de "reconnaissance » à travers un travail lexicographique de haute précision.

\section{La production} néologique n'est pas en elle-même problématique si, et seulement si, elle met en lumière des registres de signification qu'un mot ne prendrait pas en compte. Si le créateur, par son néologisme, veut seulement faire parler de sa création, alors l'opération demeure stérile. précisément des verbes : vouloir, pouvoir, oser, devoir au sens moral et, enfin, devoir au sens de contrainte. Leur interdépendance est d'ailleurs représentée géométriquement dans un pentagramme en ce qu'elle constitue « la logique de la langue » en comparaison aux axiomes de la géométrie. En plus d'être des équivalents d'axiomes, ces catégories sont « [...] des passions, des affects ou des souffrances saisis au vol et contraints à se fixer dans une forme grammaticale » (Ibid., p. 55). En outre, écrit l'auteur, « comme tous les verbes, ils peuvent être conjugués, et ainsi, personnalisés » (Ibid., p. 55). Ces catégories ont la particularité de ne s'appliquer qu'à l'humain. Une formule de l'auteur nous permet encore d'aller un pas plus loin en établissant un lien entre la critique de la psychopathologie des failles et la négativité-positivité qui se donne dans le langage des verbes pathiques : «[...] l'être humain, dans l'anthropologie pathique, se présente dès le début comme insuffisant, immature, indéterminé, défectueux ou impuissant, à la recherche d'une plénitude et d'un changement, temporel et non pas éternel, en tout cas pas comme l'Être même. Il n'est pas un être ou une chose assigné à un 'il y a', mais au contraire celui ou cela qui devient ou veut, ose, peut ou doit 'devenir' » (Ibid., p. 55) ${ }^{20}$. L'humain se dit donc dans la contrainte de ces auxiliaires comme dans celle des formes admises de sa langue, à la voix passive ou active ${ }^{21}$.

La démarche clinique gagne en intelligibilité si elle prend au sérieux la façon dont les mots jouent dans l'expression des patients, dans les tournures produites lors de la rencontre interhumaine, dans cette grammaire subjective que nous avons évoquée. Dans cette perspective, l'analyse étymologique et lexicographique pourrait, elle aussi, prendre les mots au sérieux en ce qu'ils contiennent la problématique en question ${ }^{22}$ alors que la conceptualisation scientifique - quelquefois avide de néologismes ${ }^{23}$ - écrase parfois les registres de significations que les mots renferment. Or, avant d'être des concepts, les mots sont avant tout bel et bien des mots, au fond plus riches de sens. Le concept peut trahir la complexité des significations que les hommes ont déposées dans la langue et qui s'est sédimentée au fil de l'histoire. Cependant, si l'on accorde au verbe « signifier» toute son importance dans le dispositif clinique tant dans son acception transitive qu'intransitive, convoquant toujours doublement le(s) message(s) et son (ses) 
interprète(s) dans une dimension d'adresse à l'autre, alors il nous faut aussi nous méfier du risque d'être envoûtés par le sens et la nécessité de sa production.

\subsection{Ouïr avant d'entendre, voir avant d'observer : l'en-deçà du sens}

La rencontre de Debuyst et de Demaret semble tracer une voie qui partirait de l'ethos pour aboutir à l'éthique. Cette dernière reposerait sur une lecture de la pathologie dans une visée adaptative à travers ce que nous proposons d'appeler la «normativité subjective ». L'éthique clinique qui s'esquisse doit être comprise comme une ouverture aux proto-pensées ou aux jeux de langage alternatifs, ou encore à la reconnaissance de l'intentionnalité spécifique sous l'épaisseur du sens. En tant que clinicien - mais aussi « en tant qu' animal » pourrions-nous dire -, il s'agit au fond d'ouïr avant que l'activité d'écoute ne se complique des tensions inhérentes au problème complexe de la signification. Si la criminologie clinique à visée compréhensive se doit nécessairement de thématiser et de recourir au jeu de la signification, nous suggérerions volontiers un déplacement. Celui-ci consisterait à se positionner en-deçà, ou sous l'épaisseur de la signification clinique. Dans une perspective qui considérerait que l'action n'a d'autre but qu'elle-même - ou qu'elle recouvre l'ensemble de tous les buts tant imaginables qu'inimaginables -, en tant que téléologie interne et autosuffisante, il s'agirait peut-être "d'ouïr sans (trop) entendre $»^{24}$. Dans cette position originale, le clinicien accorderait au langage la faveur de sa puissance sonore et poétique, tout en gardant à l'esprit que la séparation de ces activités de l'ouïe et de l'entendre constitue un impossible phénoménologique - autrement dit, un devoir toujours empêché. Le verbe pathique qui se donnerait à écouter et à entendre dans ses tensions spécifiques et singulières de la grammaire subjective, constituerait déjà un détachement de ce fond sonore comme un (pré)objet ou un « système proto-pathique $»^{25}$ sans fioritures sémantiques ou esthétisme symbolisant, que le travail d'interprétation pourrait lui prêter trop rapidement. Les verbes pathiques sont néanmoins des auxiliaires et, comme le dit von Weizsäcker, « [...] ils ne font qu'aider sans pouvoir rien produire. Ils sont un bâton de marche, pas la promenade » (von Weizsäcker, 2011, p. 59).
24 Restant dans le contexte du « jeu », et même du « jeu de mots ", nous pouvons retrouver une intuition de génie de cette attitude quasiment surréaliste dans le texte " Ouï-dire » de Raymond Devos (1991).

25 Ce néologisme résulterait d'une articulation du concept de proto-pensée avec celui de système de catégories pathiques. 
26 II ne faut bien entendu pas confondre cette prise de position méthodologique avec le projet a-théorique du DSM. Cette proposition de suspendre les a priori théoriques correspond plutôt au projet phénoménologique husserlien de l'Épochè. Celui-ci, dans lequel s'inscrivent intuitivement tant Debuyst que Demaret, réfute en quelque sorte la possibilité d'une approche fondamentalement dépourvue de toute forme de théorie. Selon l'épistémologie poppérienne, l'idée est de favoriser la reconnaissance de positions implicites qui innervent toute connaissance. L'on citera cet autre épistémologue fécond, Gaston Bachelard : « II faut donc que le savoir s'accompagne d'un égal oubli du savoir. Le non-savoir n'est pas une ignorance mais un acte difficile de dépassement de la connaissance » (Bachelard, 1957, p. 15, mis en italique par nos soins).
S'il s'agit d'ouïr sans entendre, il est sans doute également question de voir avant d'observer. Ce dernier point nous permettant, toujours dans un contexte phénoménologique, de boucler la boucle et de retomber in concreto dans la principale des préoccupations de l'éthologue. Cette position nous permet, en outre, de reprendre à nouveaux frais notre réflexion sur le primat de la surprise inhérent à la rencontre clinique, au détriment de la recherche effrénée de signification. Donnons la parole à l'un de nos deux maîtres : « [...] jeune étudiant en médecine, désireux de commencer la formation de psychiatre, nous avions ouvert un manuel trouvé par hasard dans les rayons d'une librairie, et dont l'auteur conseillait, dans les premières pages, de partir à la rencontre des malades mentaux, sans aucune formation théorique préalable. C'est ce que nous avons fait, commençant 'naïvement' notre carrière d'interne en psychiatrie en allant voir les malades : les catatoniques, maniaques, paraphrènes et autres grands délirants dans les asiles. L'éthologie, elle aussi, suppose une certaine expérience préalable de l'observation naturaliste sur le terrain. Nous inciterons donc ceux de nos lecteurs qui n'ont encore rien lu en éthologie et en psychiatrie, d'aller eux aussi à la découverte, dans la nature et dans les instituts psychiatriques, s'ils le peuvent, avant de lire, et de ne pas procéder inversement, comme cela se fait généralement. La démarche que proposait Baruk (1950), auteur de ce premier manuel de psychiatrie que nous avons eu dans les mains, était fondamentalement éthologique : observer, sans a priori, avant toute chose » (Demaret, 1979, pp. 17-18).

Cette proposition paradigmatique de Demaret, prône donc une position a-théorique princeps ${ }^{26}$. Si nous y souscrivons radicalement, nous estimons même utile de préciser que, plus encore qu'observer, il est sans doute nécessaire, dans un premier temps - qui marquerait la « position originelle » du criminologue clinicien - de voir. La nécessité de recourir au voir pose un enchevêtrement complexe entre les positions de passivité et d'activité. Ce que nous souhaitons mettre en exergue dans le fait de précéder l'observation par le voir est de rendre à la démarche du clinicien sa position absolument naïve. L'on pourrait dès lors « voir » (ou « entendre ») dans la notion d'observation une attitude plus active que dans celle de voir. Il n'en est rien, selon nous. L'art de la clinique réside précisément dans cette 
faculté à activer, en quelque sorte maximiser, le voir. Il s'agit de transmuter l'acte de voir, de le faire passer d'une position a priori passive vers une attitude résolument active. Ce qui différencie, dès lors, le voir de l'observation, et ce qui donne au premier de ces termes une position plus fondamentale et plus antérieure, est le recours à l'a priori théorique. Ce dernier contamine assurément moins le clinicien occupé à voir que celui qui observe, muni de son éthogramme - et dès lors le comportement qu'il s'apprête à observer.

Le gain d'intelligibilité, reposant sur les notions d'adaptation, de jeu et de la dialectique ouirr-voir, est au fond principalement clinique et méthodologique. Il retrouve la thèse épistémologique de Debuyst qui, il y a plus de trente ans, s'interrogeait sur les modes de production de la connaissance clinique et criminologique.

\section{Conclusion}

Les références à l'éthologie n'ont pas manqué d'indiquer la richesse de matériaux susceptibles de nourrir la réflexion d'une criminologie clinique dynamique. Debuyst et Demaret, dans le dialogue uchronique que nous avons proposé dans cet essai, nous offrent matière à développer une diatribe raisonnée à l'encontre d'une psychopathologie des failles. Cette critique invite le clinicien à raisonner contre l'aveuglement qui promeut la négativité et à soutenir une lecture positive et adaptative tant de la délinquance que de la pathologie, au sens où la première pourrait constituer non seulement une pathologie du langage mais aussi l'expression d'un langage pathologique. Il pourrait s'agir, d'une part, d'une restriction et/ou non-actualisation des possibles et, d'autre part, d'un fonctionnement singulier à mettre au jour comme monde propre.

Les verbes et la grammaire subjective deviennent sources d'une attention renouvelée sans néanmoins se laisser séduire par le problème de la signification ; du moins sans réduire nos démarches compréhensives à la production de celle-ci. De même, lorsque l'acte délinquant tranche dans un processus qui apparaît sans horizon sémantique immédiat, laissant perplexe le clinicien obsédé par le sens comme rapport signifiant/ signifié, le redéploiement des possibles non advenus peut 
27 Et de penser, à la suite des travaux de Despret (2009), l'homme avec l'animal. inaugurer un nouvel espace prometteur, au-delà du tragique de l'absolu de l'acte : Et s'il en avait été autrement? Quelles auraient pu être les issues différentes? Le " pathique » nous guiderait alors en nous laissant porter par la grammaire subjective qui pourrait conjuguer autrement, en une nouvelle liberté jouant dans les mots de nos patients dits délinquants, mots à la fois porteurs d'une reprise de l'acte comme relecture, mais tournés plus fondamentalement vers une possible métamorphose de soi, d'un être en tension entre ce qu'il était et ce qu'il peut peut-être devenir. De l'ethos à l'éthique, dans un aller et retour constant, nous serons forcés de considérer l'animal dans l'homme comme l'homme dans l'animal ${ }^{27}$, sans pouvoir penser en dehors de tout effort nécessaire de (re)contextualisation et sans nous laisser envoûter par les sirènes trop évidentes de l'inadaptation. La criminologie clinique nous semble gagner en intelligibilité grâce aux affinités de pensée observées entre Albert Demaret et Christian Debuyst. Les hypothèses que notre sens partagé du travail clinique a fait naître, seraient justifiées si cette phénoménologie des organes sensoriels esquissée pouvait trouver ses conséquences dans la pratique clinique.

Adam, Christophe, (2015), Psychopathologie et délinquance, Bruxelles, Bruylant.

Bachelard, Gaston, (1957), La poétique de l'espace, Paris, PUF, 2004.

Binswanger, Ludwig, (1963), Being-in-the-World, New York, Basic Books.

Bouveresse, Jacques, (1995), Langage, perception et réalité, T. 1. La perception et le jugement, Nîmes, Éditions Jacqueline Chambon.

Bouveresse, Jacques, (2004), Langage, perception et réalité, T. 2. Physique, phénoménologie et grammaire, Nîmes, Éditions Jacqueline Chambon.

CAnguilhem, Georges, (1966), Le normal et le pathologique, Paris, PUF.

Coren, Stanley, (2010), Foreword, In Fine, A.H. (Ed.) Handbook on animalassisted therapy: Theoretical foundations and guidelines for practice, pp. xv-xviii, Waltham, Massachusetts, Academic Press.

Debuyst, Christian, (1977), Le concept de dangerosité et un de ses éléments constitutifs : la personnalité criminelle, in Debuyst, C., (2009), Essais de criminologie clinique. Entre psychologie et justice pénale (Textes choisis par C. Adam et F. Digneffe), Bruxelles, Larcier.

Debuyst, Christian, (1985), Modèle éthologique et criminologie, Bruxelles, Mardaga.

Debuyst, Christian, (1986), Questions d'épistémologie : l'étude du comportement délinquant et ses implicites, in Debuyst, C., (2009), Essais de 
criminologie clinique. Entre psychologie et justice pénale (Textes choisis par C. Adam et F. Digneffe), Bruxelles, Larcier.

DeBuyst, Christian, (2009), Essais de criminologie clinique. Entre psychologie et justice pénale (Textes choisis et présentés par Christophe Adam et Françoise Digneffe), Bruxelles, Larcier.

Debuyst, Christian, (2014), Criminologie clinique. Un passage par Wittgenstein, Bruxelles, Larcier.

De GreefF, Étienne, (1947), Les instincts de défense et de sympathie, Paris, PUF.

Demaret, Albert, (1979), Éthologie et psychiatrie, Bruxelles, Margada, 2014.

Demaret, Albert, (1991), La psychiatrie évolutionniste, Acta Psychiatrica Belgica, 91(4-5), 197-231.

Demaret, Albert, (1994), Le divan naturel, In L'Homme : la psychanalyse avait-elle raison?, pp. 109-154, Grenoble, Éditions La pensée sauvage.

Despret, Vinciane, (2009), Penser comme un rat, Sciences en questions, Versailles, Quae.

Devos, Raymond, (1991), Ouï-dire, In Matière à rire, pp. 81-82, Paris, France Loisirs.

Dummetт, Michael, (1991), Thought and Perception: The view of two philosophical innovators, In Frege and Other Philosophers, pp. 263-288, Oxford, Clarendon Press.

Englebert, Jérôme, (2013), Psychopathologie de l'homme en situation, Paris, Hermann.

ENGLEBERT, Jérôme, (2015), A new understanding of psychopathy: The contribution of phenomenological psychopathology, Psychopathology, 48(6), 368-375.

Englebert, Jérôme, (2016), Mad, bad or adapted? Psychopathology of psychopaths, In Stanghellini, G., Broome, M., Fernandez A., Fusar-Poli, P., Raballo, A., Rosfort R. (Eds) Oxford Handbook of Phenomenological Psychopathology, Oxford, Oxford University Press.

Englebert, Jérôme, Adam, Christophe, (2016), La « personnalité antisociale ", antithèse de la psychopathologie, Déviance et Société, in press.

Englebert, Jérôme, Gauthier, Jean-Marie, (2011), Éthologie et psychiatrie : hommage au travail du Docteur Albert Demaret, Acta Psychiatrica Belgica, 111(4), 8-12.

Englebert, Jérôme, Follet, Valérie, (2014), Essai de psychopathologie éthologique, in Demaret A., Éthologie et psychiatrie, Bruxelles, Mardaga, pp. 165-231.

KinaBle, Jean, (1998), Transgression et passage à l'acte psychopathique, In Jonckheere, P. (Ed) Passage à l'acte, Bruxelles, De Boeck Université, pp. 105-145.

Kinable, Jean, (1990), Le sens de la délinquance, In Acteur social et délinquance, Liège, Mardaga, pp. 380-382. 
Merleau-Ponty, Maurice, (1964), Le visible et l'invisible, Paris, Gallimard.

Minkowski, Eugène, (1966), Traité de psychopathologie, Paris, Les empêcheurs de penser en rond, 1999.

Price, John, Gardner, Russell, Wilson, Daniel, Sloman, Leon, Rohde, Peter, ERICKSON, Mark, (2007), Territory, rank and Mental Health: the history of an idea, Evolutionary Psychology, 5(3), 531-554.

PricE, John, (2016). Territoire, rang social et humeur dépressive : L'histoire d'une idée. In Englebert, J., Follet, V. (Eds) Adaptation : Essai collectif à partir des paradigmes éthologiques et évolutionnistes, pp. 191-215, Paris, MJW Fédition.

Ricceur, Paul, (2004), Parcours de la reconnaissance, Paris, Stock.

SAmi-Ali, (1980), Le banal, Paris, Gallimard.

von WeIzsäcker, Viktor, (2011), Pathosophie (traduit de l'allemand par J. de Bisschop, M. Gennart, M. Ledoux, B. Maebe, C. Mugnier \& A.-M. Norgeu), Grenoble, Million.

Wittgenstein, Ludwig, (1953), Recherches philosophiques, Paris, Gallimard, 2005. 